\title{
Tuberculosis: an old world disease providing new world challenges in NSW
}

\author{
Guy B. Marks ${ }^{\mathrm{A}, \mathrm{E}}$, Amanda Christensen ${ }^{\mathrm{B}}$, \\ Vitali Sintchenko ${ }^{\mathrm{C}, \mathrm{D}}$ and Chris Lowbridge ${ }^{\mathrm{B}}$ \\ ${ }^{\mathrm{A}}$ Department of Respiratory Medicine, Liverpool Hospital \\ ${ }^{\mathrm{B}}$ Health Protection NSW \\ ${ }^{\mathrm{C}}$ Centre for Infectious Diseases and Microbiology, \\ Westmead Hospital \\ ${ }^{\mathrm{D}}$ Sydney Institute for Emerging Infectious Diseases \\ and Biosecurity, The University of Sydney \\ ${ }^{\mathrm{E}}$ Corresponding author.Email: guy.marks@sydney.edu.au
}

Tuberculosis (TB) is a complex, difficult disease from both public health and clinical perspectives. Problems are the norm, rather than the exception. The NSW TB Control Program consists of a network of highly experienced physicians, nurses, laboratory and public health personnel, working closely to manage this ongoing threat to public health. The following four case studies present issues encountered in managing TB in New South Wales (NSW).

In the first example, Michail draws our attention to the challenges of adverse drug reactions in the management of TB. Early detection, which requires vigilance and judicious monitoring, is critical for reducing the risk of harm. The serious nature of TB, the limited range of effective drugs available and the difficulty in identifying a single causative drug from a multi-drug regimen means that permanent drug cessation or replacement is not a simple option in most cases. Careful consideration is required in deciding if, when and how to re-introduce drugs after suspected adverse drug reactions. While published guidelines offer some assistance, there is enormous variation in the clinical circumstances surrounding each case. Hence, clinical experience and judgment is required. Sometimes the combined experience of several clinicians, gathered by informal consultation or by way of clinical case conferences, is needed to reach decisions on more complex cases. Michail's article highlights the importance of specialised clinical management of TB to optimise patient safety.

Experience, expertise and judgment are all relevant to public health, as well as the clinical management of TB, as highlighted in the two examples by Banner and Fisher et al. In the public arena, TB remains an emotive and poorly understood disease. Fear, anxiety, distrust and suspicion are easily aroused when people are informed they may have had contact with people with TB. On the other hand, failure to detect and treat latent TB infection (LTBI) in contacts of people with TB may result in avoidable cases of TB occuring in those contacts; this may have disastrous consequences if those contacts are young children or infants. Good management is complex and requires balancing the public's right to know with the patient's right to privacy, and the need to protect those at risk with the need to avoid spreading alarm by unnecessary screening. We have imperfect tools for estimating risk in this context and much of the difficulty in managing TB contact investigations arises from the need to make decisions in the presence of substantial uncertainty. For example, the lack of specificity of tests for LTBI means that testing patients at very low risk for LTBI, either by tuberculin skin test or by interferon-gamma release assay, will identify many false positive cases, causing false alarm and, possibly, the initiation of an unnecessary course of treatment. This highlights the importance of carefully selecting those to be screened for LTBI. The episodes reported here draw attention to the importance of pooling our statewide experience in managing complex public health problems in TB care and applying a considered and protocol-driven approach to management.

Vogelnest's report on TB in animals reminds us that, no matter how much we think we know collectively, we should always expect the unexpected in managing TB. While human TB mainly affects humans, other animals can be infected (usually by humans) and can be a source of infection for other animals and humans. Fortunately no cases of active TB in humans have arisen from the recent cases at an Australian zoo. ${ }^{1}$

The outcomes of the NSW TB Control Program have been good for many years. The incidence of the disease is low, deaths are uncommon, ${ }^{2}$ local transmission is limited ${ }^{3}$ and relapse after treatment is very rare. ${ }^{4}$ Despite these positive outcomes the following case studies demonstrate that there is no room for complacency. The complex and unpredictable nature of $\mathrm{TB}$ means that we must retain vigilance, programmatic management, and our collective clinical and public health expertise, to ensure that the gains of the last 20 years are consolidated and that further progress is made. 


\section{References}

1. Stephens N, Vogelnest L, Lowbridge C, Christensen A, Marks GB, Sintchenko V et al. Transmission of Mycobacterium tuberculosis from an Asian elephant (Elephas maximus) to a chimpanzee (Pan troglodytes) and humans in an Australian zoo. Epidemiol Infect 2013; 141(7): 1488-97.

2. Lowbridge C, Christensen AJ, McAnulty JM. EpiReview: Tuberculosis in NSW, 2009-2011. N S W Public Health Bull 2013; 24(1): 3-9.
3. Gallego B, Sintchenko V, Jelfs P, Coiera E, Gilbert GL. Three-year longitudinal study of genotypes of Mycobacterium tuberculosis in a low prevalence population. Pathology 2010; 42(3): 267-72. doi:10.3109/00313021003631346

4. Dobler CC, Crawford ABH, Jelfs PJ, Gilbert GL, Marks GB. Recurrence of tuberculosis in a low incidence setting. Eur Respir J 2009; 33(1): 160-7. doi:10.1183/09031936.00104108 